\title{
Norma y unicidad del sistema técnico en la producción de alimentos en Argentina
}

\author{
Technical system standard and unicity in Argentina's food production
}

\author{
Silvia Inés Busch \\ silviabusch@gmail.com \\ CONICET/ Instituto de Geografía / Facultad de \\ Filosofía y Letras / Universidad de Buenos Aires, \\ Argentina
}

Recepción: 24 Febrero 2020

Aprobación: 07 Junio 2020

Publicación: 02 Noviembre 2020

Cita sugerida: Busch, S. (2020). Norma y unicidad del sistema técnico en la producción de alimentos en Argentina. Geograficando, 16(2), e072. https:// doi.org/10.24215/2346898Xe072

\begin{abstract}
Resumen: El período contemporáneo se caracteriza por la constitución de un medio técnico-científico-informacional sobre territorios cada vez más vastos. Con el alargamiento de las divisiones territoriales del trabajo de las grandes empresas se imponen nuevas formas de cooperación y control. En este trabajo analizamos la creciente mediación de las normas en los circuitos de la economía urbana orientados a los alimentos de rápida preparación con sede en la metrópoli de Buenos Aires. Para hacerlo estudiamos las transformaciones en la regencia en los circuitos y el fenómeno técnico como condición de posibilidad de la normatización del territorio. Por último, indagamos las implicancias de este proceso en las relaciones entre actores hegemónicos y subordinados.
\end{abstract}

Palabras clave: Norma, Circuitos de la economía urbana, Medio técnico-científico-informacional, Uso corporativo del territorio, Fenómeno técnico.

\begin{abstract}
The contemporary age is characterized by the constitution of a technical-scientific-informational medium over increasingly larger territories. New forms of co-operation and control are imposed due to expanding large-company labor territory divisions. In this paper, the growing standard mediation in urban economy circuits of fast-preparation foodstuffs located in the Buenos Aires metropolis is analyzed. To this end, regency transformation in circuits and the technical phenomenon as a condition of possibility for territory standardization have been studied. Lastly, we discuss implications of this process on relations between hegemonic and subordinate actors.
\end{abstract}

Keywords: Standard, Urban economy circuits, Technicalscientific-informational medium, Corporate use of territory, Technical phenomenon.

\section{INTRODUCCIÓN}

El período contemporáneo se caracteriza por la constitución de un medio técnico-científicoinformacional sobre territorios cada vez más vastos (Santos, 1993). Aunque la génesis de esa nueva configuración territorial se encuentra en la Segunda Guerra Mundial, gracias a las tecnologías de comunicación e información y a la expansión del consumo, los contenidos técnico-científicoinformacionales alcanzan a los países periféricos hacia 1970 (Santos, 1972). En cada formación 
socioespacial difusión de esos vectores encuentra sistemas de acciones y objetos preexistentes, y se genera una mayor diferenciación en el medioambiente construido (Santos, 2013). La selectividad en los procesos de modernización constituye a la geografía de ese grupo de países.

El conjunto de técnicas que emerge a mediados del siglo XX permitió la disociación geográfica de actividades más grande que había conocido la historia (Santos, 1972). Con el alargamiento de las divisiones territoriales del trabajo de las grandes empresas vemos crecer sus sistemas de control. Como ha señalado Milton Santos (1993, p. 46), "la profundización de la división del trabajo impone formas nuevas y más elaboradas de cooperación y control”. Variable ascendente del período, la organización "es causa y consecuencia del aumento y la complejidad de las instituciones, de las normas y las fiscalizaciones" (Silveira, 2016, p. 85). De este modo, el período de la globalización se caracteriza por una mediación cada vez mayor de las normasen los sistemas de acciones y en los sistemas de objetos.

Entendidas como eventos productores de racionalidad (Silveira, 2006), las normas constituyen un acontecer jerárquico. Santos (2000, p. 141) señala que estos eventos son el resultado de la tendencia a la racionalización de las actividades y se realizan bajo una dirección proveniente de otro lugar; se trata "de un cotidiano dirigido por una información privilegiada, una información que es secreto y es poder" ${ }^{1}$. Podemos decir entonces que las normas son tanto causa como consecuencia de la tendencia a la racionalización de actividades.

El predominio del acontecer jerárquico conlleva la subordinación creciente de las formas menos capitalizadas de producción, distribución y comercialización. En los países periféricos, como causa y resultado de las sucesivas modernizaciones, se conforman, en las ciudades, dos circuitos de producción, distribución y consumo de bienes y servicios: circuito superior y circuito inferior de la economía urbana (Santos, 2008).Las variables principales que permiten distinguirlos son el capital, la tecnología y la organización. Estos se constituyen relacionalmente y están atravesados por diferencias de poder. Las lógicas del circuito superior subordinan empresas menores que poseen grados más bajos de tecnología y capital, y que conforman la porción marginal del circuito superior (Silveira, 2016).

En este artículo analizamos la creciente mediación de las normas en los circuitos de la economía urbana orientados a los alimentos de rápida preparación con sede en la metrópoli de Buenos Aires. Interrogamos los sistemas de acciones y de objetos que constituyen a esa mediación; las relaciones de poder que atraviesan la constitución y el dominio de las normas, la relación entre la difusión de normas y la densificación del medio técnico-científico-informacional, y sus efectos en el devenir de las formas menos capitalizadas de producción. En el ramo de los alimentos, estudiamos la producción y comercialización de empanados de pollo congelado, vegetales congelados y sopas y condimentos para carnes con base en vegetales deshidratados. En la medida en que las topologías de las firmas que participan de esos circuitos espaciales de producción ${ }^{2}$ se extienden a lo largo del territorio nacional, el estudio alcanza diversos lugares de la formación socioespacial argentina y especialmente su área concentrada. ${ }^{3}$

En el primer apartado indagamos los cambios en la regencia en los circuitos de la economía urbana orientados a los alimentos de rápida preparación. En segundo lugar, estudiamos el fenómeno técnico como condición de posibilidad en la normatización del territorio. Por último, indagamos las implicaciones del aumento de las normas en las relaciones entre actores hegemónicos y subordinados.

\section{Mudanzas en la regencia técnico-Política de los Circuitos de la economía URBANA EN ALIMENTOS}

En la década de 1990, la política pública desarrollada desde el Estado argentino profundizó el modelo financiero y de ajuste estructural que tendió a la privatización de prácticamente la totalidad de las empresas 
estatales y forjó la apertura de la economía a los flujos internacionales de bienes y capitales y la desregulación de múltiples mercados (Aspiazu y Schorr, 2010). Esos contenidos normativos para liberar las finanzas configuraron "una división interna del trabajo que tiende a fortalecer al circuito superior en la red urbana nacional, es decir, a grandes grupos económicos y financieros" (Parserisas, 2018, p. 64). Entre otros hechos, creció el número de empresas globales alimenticias que operan en el país. Estos grupos reforzaron la orientación exportadora de la producción de alimentos (Bisang y Gutman, 2005) e indujeron reestructuraciones en la actividad. Como explican Gutman y Gorenstein (2005, p. 7), imprimieron "una convergencia del sector a los parámetros operativos internacionales".

El aumento del control de las grandes empresas globales sobre el sistema productivo de alimentos a escala mundial (Gutman y Gorenstein, 2005) se basa en innovaciones organizacionales y de gerenciamiento (Silveira, 2009; Silva, 2011). Entre las innovaciones desarrolladas por las empresas del circuito superior orientado a los alimentos de rápida preparación-junto con la expansión de sus divisiones territoriales del trabajo-se destacan la centralización en la toma de decisiones y la tercerización de funciones. Esta última abarca tanto la contratación de servicios cualificados que acompañan las nuevas formas organizacionales (consultorías en normas, empresas que las certifican, agencias de publicidad, estudios contables y jurídicos) como la tercerización de tareas productivas y de servicios banales.

En las grandes firmas, el control sobre cada una de las sedes ante la expansión de sus divisiones territoriales del trabajo particulares se sostiene cada vez más sobre normas internas; "las normas de las empresas son hoy una de las locomotoras de su actuación y de su rentabilidad” (Santos, 2000, p.193).En empresas como Unilever o Nestlé vemos crecer el control de las casas matrices sobre los lugares donde extienden sus topologías. Ese aumento de la normatización se traduce en la complejización de funciones en sus sedes de regencia y en la subordinación técnico-política de cada una de las sedes que conforman sus divisiones territoriales del trabajo a lo largo del país. En la planta de Magdalena ${ }^{4}$ de Nestlé, una trabajadora en el área de Mejora Continua señalaba que desde las oficinas de Suiza envían instrucciones: "en 2010 empezaron a implementar objetivos del negocio, a través de los cuales evalúan el alineamiento de objetivos, vinculados con el cumplimiento de las normas internas" (trabajadora en el sector de Mejora Continua en la planta de Nestlé en Magdalena, comunicación personal, 3 de febrero de 2017).

Las normas internas están orientadas a aumentar la productividad y también a cumplimentar las normas externas. Podríamos decir que con la expansión de los circuitos espaciales de producción se trastocan también los círculos de cooperación ${ }^{5}$ que entreteje el conjunto de actores que participa de la actividad. En ese sentido, la división del trabajo en la regulación de la producción de alimentos se ha complejizado en las últimas décadas. La internacionalización de las normas que atañen a la elaboración y comercialización de los alimentos se ha profundizado a partir de la creación de la Organización Mundial del Comercio (OMC) en 1995 (Marichal, 2013). Esa organización constituye una novedad de la década de 1990, una "instancia mundial de elaboración, fiscalización y sanción de reglas del comercio internacional” (Silveira, 1999, p. 248). En la actualidad, el principal cuerpo de normas mundiales relacionado con la alimentación es el Codex Alimentarius, regulación elaborada conjuntamente por la Organización para la Alimentación y la Agricultura (FAO) y la Organización Mundial para la Salud (OMS). Este tipo de organizaciones gubernamentales constituyen regímenes de regulación internacional; están controladas desde sus orígenes por los países centrales, aunque fueron creadas por los Estados, "disponen de sus aparatos, de sus redes de influencia, y de sus medios de acción propios” (Arroyo, 2001, p. 43).

La funcionalización de las tendencias a escala mundial en las políticas desarrolladas desde el Estado nacional argentino se ha profundizado en las últimas décadas. Marichal (2013) ha mostrado el proceso de internalización de las normativas internacionales sobre alimentación en el cuerpo de leyes nacionales. En 1994 se incorporaron los resultados de la Ronda Uruguay de Negociaciones Comerciales Multilaterales, que aplicó por primera vez normas comerciales internacionales a los productos agrícolas (Marichal, 2013). Desde el comienzo de su funcionamiento, Argentina forma parte de la OMC. En 1999 se reafirmó que el 
Código Alimentario Argentino es la regulación fundamental del sistema, a la cual debe incorporarse toda la normativa vigente que haga a la elaboración, distribución y comercialización de alimentos (Marichal, 2013). En su conjunto, se trata del desarrollo de mecanismos jurídicos corporativos "que se inmiscuyeron (y aún se inmiscuyen) en el modo de producción jurídico estatal, proporcionando la expansión de la lógica corporativa" (Mendes, 2005, p.169).

Con todo, desde la década de 1990 también es creciente la creación de normas de calidad en ámbitos privados (Ghezán, 1999), que luego buscan imponerse en los ámbitos de negociación global. En ese sentido, entre los actores que participan del circuito superior de la economía urbana orientado a los alimentos de rápida preparación, las empresas globales se caracterizan por participar de los procesos de construcción de esas normas. Es destacable la posición de Nestlé, que junto con Coca-Cola participó de la construcción de las normas PAS 220. Además, todas las plantas de Nestlé en el país han alcanzado la certificación de las normas ISO 14001 (medio ambiente) y OHSAS 18001 (salud y seguridad ocupacional). La planta de Magdalena fue la primera que logró esas certificaciones en noviembre de 2007 y en 2015 aprobó la norma FSSC22000.La posición de los actores globales y los círculos de cooperación que entretejen llevan a que se constituyan en pioneros en el cumplimento de las normas más sofisticadas.

La interiorización de las normativas internacionales llevó a una introducción de innovaciones organizacionales, tanto en el interior de la empresa agropecuaria como en su vinculación con otros agentes (Ghezán, 1999). La difusión de las normas y de la estandarización supone una mayor complejidad en la organización del circuito espacial de producción, y en las instituciones y actores que participan de él. Una empresa global como $\mathrm{BRF}^{6}$ participa de organizaciones internacionales como el International Poultry Council (IPC) y la International Meat Trade Association (IMTA) (Director Corporativo de BRF en San Pablo, Brasil, comunicación personal, 28 de junio de 2017).

El propósito del IPC es "desarrollar y recomendar políticas que afectan nuestras industrias y promover un entendimiento común y global” (IPC, 2018). A medida que se profundiza la inserción del país en la economía internacional y se difunde la normatización del territorio, vemos una expansión de los círculos de cooperación. Aunque creadas en diferentes niveles geográficos y políticos, "son las normas globales, inducidas por organismos supranacionales y por el mercado, las que tienden a configurar a las demás. Y las normas del mercado tienden a configurar las normas públicas" (Santos,1999, p. 12).

\section{FENÓMENO TÉCNICO Y NORMATIZACIÓN DEL TERRITORIO}

Aunque las normas pertenezcan al dominio de la política (Santos, 2000), las relaciones entre políticos y expertos median sus procesos de construcción (Winickoff y Bushey, 2010). Así, los organismos internacionales recurren crecientemente al conocimiento científico para legitimar y construir las normas (Marichal, 2013). De igual modo, las normas desarrolladas en el ámbito privado se fundan en conocimiento científico: para los productos Knorr de Unilever se estableció que los agricultores que proveen a la marca deben cumplir con los lineamientos establecidos en el Código de Agricultura Sustentable de Unilever, que es el resultado de un trabajo en conjunto entre agricultores, consultores y expertos en sustentabilidad. La progresiva integración entre la ciencia y la técnica (Ellul, 1968; Simondon, 2007) lleva a que las innovaciones dependan cada vez más de aquello que Milton Santos (1972, p. 256) llamó "la invención del método de invención”, es decir, de la técnica de la investigación. Una vez instituidos en normas, esos modos de proceder hacen cada vez más difícil "saber quién, en determinadas circunstancias, regula a quién en cada lugar y en cada nación” (Silveira, 2008, p.6).

En cada certificación hay un proceso técnico que es registrado y modificado según las directivas de cada norma. Al referir a los procesos de elaboración y ya no al producto, estos sistemas de control imprimen huellas en las técnicas productivas; conllevan un modo de proceder, una secuencia de órdenes sobre la organización de la producción en el interior de cada empresa. De este modo, en cada lugar, el cumplimiento de las normas 
supone un aumento de la estandarización, el intento de "resolver anticipadamente todos los problemas que puede presentar el funcionamiento de una organización” (Ellul, 1968, p. 10).

El medio técnico-científico-informacional, expresión más concreta de la globalización, va consolidándose al ritmo de lo que Santos $(1996,2000)$ identifica como tres tendencias constitutivas de la globalización: la unicidad de la técnica, la convergencia de los momentos y la unicidad del motor. Con la unicidad de la técnica, el autor enfatiza la tendencia a la unicidad en el modo de producción, que incluye cada vez un mayor número de sociedades y territorios (Santos, 1999), con la consecuente disminución de modelos técnicos disponibles y posibilidades de elección (Santos, 2000). En ese sentido, la difusión de normas tiende a la unicidad del sistema técnico. Las tecnologías de la información y de la comunicación son una condición para la realización de esa unicidad, y, al mismo tiempo, generan una progresiva convergencia de los momentos.

En el circuito vinculado a empresas automotrices, las innovaciones organizacionales vinculadas con la separación física entre etapas materiales y la gerencia fue posible gracias "a las comunicaciones y la unificación de las normas" (Donato Laborde, 2017, p. 51). La búsqueda de homogeneidad en la organización de la producción y del trabajo a través de la implementación del World Class Manufacturing en las plantas de Fiat se asienta en la elaboración de un software orientado al control tanto de la calidad como de los trabajadores (Capogrossi, 2017).

En el circuito espacial de producción de alimentos de rápida preparación, las nuevas formas organizacionales se sustentan también crecientemente en el análisis de datos. Este proceso demanda servicios, programas y dispositivos que viabilizan la circulación instantánea de informaciones de cada sede a la administración central. Como señala David Harvey (2002, p. 184), el acceso a la información y el control sobre ella "se han convertido en elementos esenciales de la coordinación centralizada de los vastos intereses de las corporaciones". Gracias a las nuevas herramientas informáticas, la información sobre proyectos, ventas y personal puede "llegar a los niveles superiores de forma instantánea, inmediata" (Sennet, 2006, p. 41).

Por lo tanto, se ha vuelto fundamental la contratación de software y servicios que actúan solidariamente con las infraestructuras y que llevan adelante las tareas de obtención, circulación, análisis e interpretación de la información. A partir de la contratación de SAP ERP de SAP en Rasic Hnos "se logró una importante reducción en los tiempos de procesos y generación de información de gestión de la empresa y la mejora del servicio al eliminar una importante cantidad de interfases" (Datamation, 2005).

Granja Tres Arroyos ha utilizado Arballon Business Software, un software desarrollado por Ardison Software \& Consulting específicamente para la industria avícola. Tanto en Unilever como en Nestlé, la unificación de las redes informacionales se dio en el marco de reestructuraciones de los grupos orientadas a aumentar la estandarización.

Ahora bien, esa información fluye en grandes distancias y de manera veloz gracias a las infraestructuras que posibilitan las tecnologías de la comunicación y de la información. En ese sentido, "la acción global se derrama sobre el conjunto de sistemas de ingeniería, sean alcanzados, sean olvidados por la modernización, coordinando el concierto de las productividades espaciales específicas de los lugares" (Silveira, 1999, p. 251).

En el período actual, el movimiento en el territorio es comandado sobre todo por flujos no obligatoriamente materiales; esto es, capitales, informaciones, mensajes, órdenes. En ese contexto, la información es "un factor de producción que permite el funcionamiento de las complejas organizaciones que demandan la instantaneidad de los comandos y de los resultados" (Silveira, 2014, p. 159). Como resultado, las informaciones sobre el contenido de las normas, mercados, innovaciones en los productos y en las técnicas productivas y cambios en los círculos de cooperación son arena de competencia entre las empresas. Se trata de informaciones estratégicas que constituyen al poder de una corporación (Santos, 2012), "para que puedan crear, por medio de aquellos lugares escogidos, un escenario global de competitividad” (Silva, 2011, p. 380).

Sin embargo, en los circuitos de la economía urbana de alimentos de rápida preparación, vemos que, junto con esa circulación altamente selectiva y jerárquica, las grandes empresas precisan también la difusión más amplia de una serie de informaciones frente al aumento de la tercerización. Cuando la producción de materia 
prima o bien otra función productiva corre por cuenta de otro actor, las firmas del circuito superior de alimentos difunden informaciones relativas al sistema técnico, de modo que sus proveedores cumplimenten los estándares exigidos por las normas, buscando una unicidad del proceso. Esta necesidad de las grandes empresas explica el aumento del asesoramiento y los cursos de capacitación para el productor. De este modo, esas informaciones se difunden del circuito superior al superior marginal y al inferior.

La empresa Nutrifrost, con sede en el municipio de Pilar en la provincia de Buenos Aires, ha desarrollado un protocolo estricto para el trabajo en las fincas que la abastecen de vegetales, el cual abarca el cuidado del suelo y del cultivo. En la producción de vegetales demandada por Unilever, esta les provee a los productores asesoramiento, semillas, químicos, fertilizantes, entrenamiento en técnicas de cultivo y organización de las cosechas. Las grandes empresas proveen o buscan que otro proporcione el entrenamiento técnico, mientras que guardan para sí la organización de la producción. En los sistemas de acciones y objetos que atraviesan el usufructo de las licencias de las marcas de genética avícola, cada laboratorio ha desarrollado un manual exclusivo de instrucciones estrictas y minuciosas acerca de los modos de proceder para el crecimiento de los abuelos ${ }^{8}$ y su reproducción. Esa secuencia de instrucciones precisas abarca la forma en la que deben ser tratados los abuelos desde el momento que son descargados del avión hasta la comercialización del pollo BB. El tipo de informaciones incluye la cantidad de comida, la graduación en la cantidad de espacio y de luz a lo largo de los días y las formas de cruzamiento entre especies que deben respetar, entre otros tantos preceptos.

En sus reflexiones teórico-empíricas en relación con los objetos técnicos, M. Akrich (1987, p. 49) dirige su atención a la red de relaciones en la que se ven envueltos, un "conjunto de relaciones entre elementos absolutamente heterogéneos" que constituye el fondo en el que el objeto técnico se inscribe. En esos "mecanismos elementales de arreglos recíprocos del objeto técnico y su ambiente" (Akrich, 1987, p. 59), en la mediación técnica, la información se vuelve clave. Así, los procesos de innovación de las empresas del circuito superior no se concretan sin la socialización de cierto tipo de informaciones a otros circuitos de la economía urbana. A través de la circulación de la información se organiza ese fondo en el que operan los objetos técnicos y la fluidez coopera en la concretización del objeto técnico.

Los actores del circuito espacial de alimentos de rápida preparación desarrollan también vínculos con instituciones públicas y privadas, orientados a intercambiar información y conocimientos, y también a promover que las difundan. Gutman, Lavarello y Grossi (2006) han destacado la fuerte interacción entre empresas privadas y el sistema público de ciencia y tecnología en el país. Las universidades forman profesionales especializados en tecnologías e ingenierías en alimentos y prestan servicios a las empresas. En la repartición de tareas del Estado nacional, además, el SENASA es el encargado de la difusión de la información relativa a las normas y el INTA, en relación con las técnicas productivas. Los cursos de capacitación y el asesoramiento otorgados por sendas instituciones colaboran de este modo en la difusión del sistema técnico a lo largo del territorio argentino, que se vuelve nodal en el contexto de la ampliación de la tercerización.

\section{ACONTECER JerárQuico EN LOS CIRCUITOS DE LA ECONOMÍA URBANA EN ALIMENTOS}

Hemos señalado que el acontecer jerárquico es la acción dirigida por una información privilegiada, que resulta de la racionalización de las actividades y cuyo poder se encuentra fuera de ese lugar (Santos, 2000). Los procesos de modernización llevan a un predominio del acontecer jerárquico en los sistemas de acciones de los países periféricos. En ese sentido, se ha señalado cómo actores globales determinan cambios en las formas de producción en ese grupo de países, condicionando la compra al cumplimiento de ciertas normas. Según Arroyo (2001), el sistema de normas que rige el vínculo entre las empresas globales y las medias y pequeñas en Brasil es diseñado por las primeras. En gran medida, el pollo consumido en el Reino Unido es importado de Tailandia y Brasil, gracias a que, en esos países, la calidad se ajusta a los estándares de industriales y comercializadores (Yakovleva y Flynn, 2004). El aumento de los estándares en el consumo en países europeos ha implicado profundas reestructuraciones en la producción hortícola tendientes a la normatización, tanto en Kenya (Asfaw et al., 2010) como en Senegal (Baglioni, 2017). Di Nucci (2010) ha 
mostrado que en la producción de bebidas gaseosas y aguas saborizadas en Argentina las grandes empresas imponen sus normas, mientras que las empresas nacionales del circuito superior marginal se ven imposibilitadas de participar de los acuerdos políticos. En este apartado, centraremos el análisis en aquellos actores, acciones y objetos que, bajo amenaza de perder la posibilidad de comercializar su producción, se ven subordinados en los procesos de normatización en el circuito espacial de producción de alimentos de rápida preparación.

Los certificados de calidad son atributos que distinguen a los actores que participan del circuito espacial de producción y que les habilitan el acceso a determinados mercados. Hemos señalado que, en los circuitos de la economía urbana orientados a los alimentos de rápida preparación, las empresas globales se caracterizan por participar en los procesos de elaboración de las normas y por promover su difusión. Otras empresas que conforman el circuito superior acceden a la certificación de las normas para lograr mercados, sin conseguir participar de su elaboración.

Entre las innovaciones organizacionales de las empresas del circuito superior orientado a los alimentos de rápida preparación mencionadas en el primer apartado, la tendencia a la tercerización de funciones productivas lleva a una profunda división del trabajo entre las empresas. Las empresas suelen contratar las instalaciones de otras frente a conflictos laborales en sus plantas o a necesidades eventuales de aumentar la producción. Como ha señalado Harvey (2002), la organización más ajustada y la centralización en la toma de decisiones se han impuesto a través de una intrincada variedad de acuerdos de subcontratación. La tercerización de funciones productivas implica una profunda transformación en la división del trabajo del circuito espacial de producción y comercialización de alimentos en las últimas décadas. En este contexto, la normatización del territorio es una condición determinante para la realización de la tendencia a la unicidad del sistema técnico.

En coincidencia con lo señalado por varios autores en otros países (Yakovleva y Flynn, 2004; Baglioni, 2017), en Argentina las empresas globales comercializadoras de alimentos también han incursionado en el desarrollo de marcas propias, con productos en gran medida elaborados por terceros (Viteri, 2003). El mercado de los productos con marcas de supermercados es importante para algunas empresas del circuito superior, pero también para algunas empresas del superior marginal. Además de comercializar los productos bajo su marca -Maglia-, Nutrifrost también produce marcas propias para Día \%, Carrefour, Jumbo, Walmart (Great Value), La Anónima, y Macro (Aro). Las marcas propias de los supermercados e hipermercados se destacan, de un lado, por tener un espacio asegurado en la góndola, y, de otro, por sus precios más bajos. Para las empresas del circuito superior marginal el ingreso y permanencia en las góndolas de los supermercados es una barrera difícil de franquear.

Para hacerlo, entre otras cosas, los productores deben someterse a la certificación de normas y a las auditorías que define cada grupo empresarial. Jumbo o Carrefour imponen condiciones para la compra de productos a sus proveedores, y aquellos que no logren cumplimentarlas perderán la posibilidad de comercializar con ellos. Un entrevistado con amplia trayectoria en empresas avícolas poco capitalizadas señalaba que no logró comercializar directamente su producción con Jumbo "porque tiene un nivel de exigencia y de calidad muy alto, tienen inspecciones en los lugares de producción” (empleado en Granja Dos Cuñados, comunicación personal, 22 de octubre de 2016). De este modo, los supermercados se vuelven actores principales en la difusión de las normas de calidad en los circuitos espaciales de producción de alimentos de rápida preparación.

Las empresas globales de comidas rápidas son también compradoras importantes para algunas empresas. La difusión de ese tipo de locales en la metrópoli de Buenos Aires constituye un canal de distribución de gran importancia para los empanados de pollo congelado. Algunas firmas extranjeras que operan en el país concentran sus locales en la metrópoli de Buenos Aires y operan bajo la forma de franquicias, entre ellos, McDonald's y Subway (Silveira, 2016). Granja Tres Arroyos provee nuggets de pollo a Mc Donalds y a Kentucky Fried Chicken, y Pampa Bistró es proveedora de Subway y Burger King. 
En la subcontratación de funciones productivas, y en la medida en que la empresa contratista adquiere una certificación, tiende a exigir nuevas condiciones a sus proveedores de insumos. Como hemos señalado, Nestlé es un actor principal en la promoción y difusión de las normativas de calidad; para hacerlo, verifica a sus proveedores mediante mecanismos de evaluación internos y externos. El grupo Unilever centraliza la aprobación de los proveedores en su casa matriz y también realiza controles de calidad en las plantas proveedoras. A las empresas globales, la estandarización del proceso productivo les posibilita ampliar la escala de acción y mantener una estructura centralizada en relación con la toma de decisiones, al tiempo que difunden a otros actores aquellas técnicas productivas estrictamente necesarias a sus procesos. Se trata de la ampliación de las verticalidades, ${ }^{9}$ que vuelven cada vez más intenso el control que se ejerce desde las casas matrices a los procesos productivos que se realizan en el país. Esta situación revela que la unicidad del sistema técnico no responde solo a la instalación de objetos técnicos sino también, y de modo creciente, a los sistemas de acción. [i]Desde la perspectiva de Milton Santos, existen puntos en el espacio que, separados unos de otros, aseguran el funcionamiento global de la sociedad y de la economía (Santos, 1999): "son vectores de una racionalidad superior y del discurso pragmático de los sectores hegemónicos, que crea un orden cotidiano obediente y disciplinado" (Santos, 2000, p. 240).

Dados sus menores grados de capital, tecnología y organización, Pampa Bistró conforma el circuito superior marginal orientado a los alimentos de rápida preparación. En esa firma, la única área en la que han contratado servicios modernos es en la certificación de normas, ${ }^{10}$ la certificación FSSC 22000 fue nodal para acceder al mercado de la Unión Europea. Además de tener que afrontar el pago para el acceso a las directivas de cada norma, de las consultorías y de las propias certificaciones, ${ }^{11}$ las empresas del circuito superior marginal deben antes realizar inversiones en equipamientos y en transformaciones en los procesos de producción. En palabras de uno de los Socios de Pampa Bistró, "los costos más importantes son los cambios que hay que hacer para que te la den" (Socio en Pampa Bistró, comunicación personal, 29 de septiembre de 2017). Entre otras inversiones en equipamiento e infraestructura orientadas a la inocuidad y la estandarización podemos mencionar la adecuación de la temperatura y el laboratorio interno. En relación con los procesos, se adecúa la tendencia a automatizar, a estandarizar, a mitigar los riesgos y a realizar análisis de peligro. Por esas razones, la normatización que atraviesa a las plantas productivas constituye también un aumento del capital fijo.

La normatización se difunde también a actores del circuito superior marginal en la producción avícola. En la elaboración de empanados de pollo congelado existe una división del trabajo entre los actores involucrados en la producción, faena y procesamiento de pollos. La tercerización del llamado "servicio de engorde" implica el crecimiento de los pollos a cargo de actores que reciben los pollitos BB, el alimento balanceado, la sanidad y el asesoramiento profesional de las empresas faenadoras (Ministerio de Economía y Finanzas Públicas, 2015). En ese contexto, las grandes firmas faenadoras imponen sus normas -el tipo de material del galpón, la forma de calefacción y de aireación, la distribución del alimento y del agua, etc.- para que los productores devengan sus proveedores.

\section{CONCLUSIONES}

A lo largo del texto hemos visto que en las últimas décadas se ha profundizado la mediación de las normas en los circuitos de la economía urbana orientados a los alimentos de rápida preparación con sede en la metrópoli de Buenos Aires. Esas empresas extienden sus topologías a lo largo del territorio nacional pero sobre todo en el área concentrada de la Argentina y, por ende, la normatización alcanza distintos lugares del país. Este proceso implica un aumento del capital fijo y circulante en los circuitos espaciales de producción, y, por lo tanto, un crecimiento dela dependencia del trabajo en relación con el capital en la formación socioespacial argentina en general y en el área concentrada en particular. 
Con la normatización se alargan también los círculos de cooperación que entretejen los actores que participan de los circuitos espaciales de producción. Estos son constituidos, por un lado, por agencias del Estado nacional, con acciones desarrolladas en el territorio argentino y con la participación en organizaciones internacionales. Por otro lado, se fortalecen también agencias privadas cuyos sistemas de acción alcanzan la escala mundial. En ese proceso, cada vez más son actores globales los que regulan buena parte de la dinámica de los territorios.

Hemos visto que, gracias a la tendencia a la convergencia de los momentos, la normatización del territorio profundiza la tendencia a la unicidad del sistema técnico. Podemos agregar entonces que la difusión de normas es una condición espacial para el alargamiento de las divisiones territoriales del trabajo y de los mercados, y, por lo tanto, para la unicidad del motor, es decir, el crecimiento de la plusvalía a escala mundial. Pues bien, para aquellos actores con menores grados de capital implica, bien un aumento de sus costos, o bien la desvalorización de su trabajo; por lo tanto, la difusión de las normas contribuye al aumento de la eficacia en la extracción de capital del circuito inferior y superior marginal al superior, a escala mundial. La mediación de las normas en los procesos productivos facilita que la unicidad del motor conlleve cada vez más concentración de capital.

\section{REFERENCIAS}

Akrich, M. (1987) Comment décrire les objets techniques? Techniques et culture, 9, 49-64. https://doi.org/10.4000 $/ \mathrm{tc} .863$

Arroyo, M. (2001) Território nacional e mercado externo. Uma leitura do Brasil na virada do século XX. Tese de Doutorado, Universidad de São Paulo, Brasil.

Asfaw, S., Mithöfer, D., Waibel, H. (2010) What Impact Are EU Supermarket Standards Having on Developing Countries' Export of High-Value Horticultural Products? Evidence From Kenya. Journal of International Food \& Agribusiness Marketing, 22:3-4, 252-276. https://doi.org/10.1080/08974431003641398.

Aspiazu, D. y Schorr, M. (2010) Hecho en Argentina. Industria y economia, 1976-2007. Buenos Aires: Siglo XXI Editores.

Baglioni, E. (2017) Labour control and the labour question in global production networks: exploitation and disciplining in Senegalese export horticulture. Journal of Economic Geography, 18(1), 1-27. https://10.1093/j eg/lbx013.

Bisang, R., Gutman, G. (2005) Acumulación y tramas agroalimentarias en América Latina. Revista de la CEPAL, 87.

Capogrossi, M. L. (2017) La disciplina tras los Sistemas Corporativos Empresariales. El World Class Manufacturing y la reorganización del trabajo en Fiat. Revista Pymes, Innovación y Desarrollo, 5(2), 31-49. Recuperado de http s://ri.conicet.gov.ar/handle/11336/56918.

Di Nucci, J. (2010) División territorial del trabajo y circuitos de la economia urbana: bebidas gaseosas y aguas saborizadas en Buenos Aires, Mar del Plata y Tandil. Tesis de Doctorado en Geografía, Universidad Nacional del Sur, Argentina.

Datamation (2005) SAP obtiene su primer implementación en el sector agroindustrial. Recuperado de http://www.dat amation.com.ar/sapobtienesuprimerimplementacionenelsectoragroindustrial1538.

Donato Laborde, M. G. (2017) Territorio y globalización en Argentina: los circuitos de la economía urbana vinculados a las empresas automotrices. Tesis de doctorado. Universidad Nacional de La Plata, Argentina. Recuperado de h ttp://www.memoria.fahce.unlp.edu.ar/tesis/te.1378/te.1378.pdf

Ellul, J. (1968) A técnica e o desafio do Século. Rio de Janeiro: Paz e Terra.

Ghezán, G. (1999) Procesos de reestructuración de la industria agroalimentaria en Argentina. El caso de hortalizas congeladas. En J. Wilkinson y R. S. Maluf (Org.) Reestructuración del sistema agroalimentario (pp. 89-108). Río de Janeiro, Brasil: RedCApa. 
Gutman, G. y Gorenstein, S. (2005) Las transnacionales alimentarias en Argentina. Dinámica reciente e impactos territoriales. Primeras Jornadas de Economia Regional Comparada, Simposio Ventajas Competitivas de los Agronegocios en el MERCOSUR, Porto Alegre. Recuperado de http://cdn.fee.tche.br/jornadas/2/E13-14.pdf.

Gutman, G. Lavarello, P. Grossi, J. (2006) La biotecnología y las industrias de ingredientes alimentarios en Argentina. J. Technol. Manag. Innov., 3(1).

Harvey, D. (2002) La condición de la posmodernidad. Buenos Aires: Editorial Amorrortu.

IPC (2018) Mission. Recuperado de http://www.internationalpoultrycouncil.com/about/mission.

Marichal, M. E. (2013) La regulación de los alimentos en Argentina. Emergencia, codificación y resistematización del Derecho Alimentario. Tesis de Doctorado en Derecho, Facultad de Ciencias Jurídicas y Sociales, Universidad Nacional del Litoral, Argentina.

Mendes, R. (2005) Território e regulacão. Espa\#o geográfico, fonte material e não-formal do dereito. São Paulo: Associa\#ão Editorial Humanitas.

Argentina. Ministerio de Economía y Finanzas Públicas (2015) Complejo Avicola, Serie Complejos Productivos. Secretaría de Política Económica y Planificación del Desarrollo, Subsecretaría de Planificación Económica, Dirección Nacional de Planificación Regional - Dirección Nacional de Planificación Sectorial.

Palacios, E. P. (2003) El complejo agroindustrial avícola argentino. Reconversión y perspectiva de inserción en el mercado regional e internacional. Revista Aportes para la Integración Latinoamericana. Recuperado de https:// revistas.unlp.edu.ar/aportes/article/view/3318.

Parserisas, D. (2018) Urbanización y finanzas en la Provincia de Buenos Aires: dinámicas contemporáneas de los circuitos de la economía urbana. Tesis de doctorado, Universidad de Buenos Aires, Argentina.

Santos, M. (1972). Dimension temporelle et systèmes spatiaux dans les pays du Tiers Monde. Tiers-Monde, 13(50), 247-268. https://10.3406/tiers.1972.1848.

Santos, M. (1993) A urbanização brasileira. São Paulo: Hucitec.

Santos, M. (1996) Metamorfoses do espa\#o habitado, Fundamentos teóricos e metodológicos da Geografia. São Paulo: Hucitec.

Santos, M. (1999) Modo de produção técnico-científico e diferenciação espacial. Território, $I V(6)$.

Santos, M. (2000) La naturaleza del espacio. Técnica y tiempo. Razón y emoción. Barcelona: Editorial Ariel.

Santos, M. (2008) Oespa\#to dividido. San Pablo: EDUSP.

Santos, M. (2012) Por uma Economia política da cidade. São Paulo: Edusp.

Santos, M. (2013) Técnica, Espa\#o, tempo (5. Ed., 1. Reimpr). São Paulo: Editora da Universidad de São Paulo.

Santos, M. (2014) Espa\#o e Método (5. Ed., 2. Reimpr.). São Paulo: Editora da Universidad de São Paulo.

Santos, M. Silveira, M. L. (2011) O Brasil. Território e sociedade no inicio do século XXI. Rio de Janeiro: BestBolso.

Sennett, R. (2006) La cultura del nuevo capitalismo. Barcelona: Anagrama.

Silva, A. B. (2011) A nova divisão territorial do trabalho brasileira e a produ\#ão de informa\#ões na cidade de São Paulo (as empresas de consultoria). En M. Santos y M. L. Silveira, O Brasil. Território e sociedade no início do século XXI. Rio de Janeiro, BestBolso.

Silveira, M. L. (1999) Um país, uma região. Fim de século e modernidades na Argentina. São Paulo: FAPESP.

Silveira, M. L. (2006) O espa\#o geográfico: da perspectiva geométrica à perspectiva existencial. GEOUSP: Espaço e Tempo, (19), 81-91. https://doi.org/10.11606/issn.2179-0892.geousp.2006.73991

Silveira, M. L. (2008) Globalización y territorio usado: imperativos y solidaridades. Cuadernos del Cendes, 25(69), 1-19.

Silveira, M. L. (2009) Finan\#as, consumo e circuitos da economia urbana na cidade de São Paulo. CADERNO CRH, 55(22), 65-76. https://doi.org/10.1590/S0103-49792009000100004.

Silveira, M. L. (2011) Território usado: dinamicas de especializa\#ao, dinamicas de diversidade. Ciência Geográfica, $X V(1)$.

Silveira, M. L. (2014) A natureza relacional dos circuitos da economia urbana. Em De Oliveira, Freire et. al (org) Geografia urbana. Río de Janeiro: Consequência. 
Silveira, M. L. (2016) Circuitos de la economía urbana. Ensayos sobre Buenos Aires y São Paulo. Buenos Aires: Editorial Café de las Ciudades.

Simondon, G. (2007) El modo de existencia de los objetos técnicos. Buenos Aires: Prometeo Libros.

Viteri, M. L. (2003) Documento $N^{\circ} 14$ : Hortalizas congeladas. Instituto Interamericano de Cooperación para la Agricultura, Secretaría de Política Económica, Ministerio de Economía de la Nación.

Winickoff, D. E., Bushey, D. M., (2010) Science and Power in Global Food Regulation: The Rise of the Codex Alimentarius. Science, Technology, \& Human Values, 35(3) 356-381. Recuperado de https://doi.org/10.1177/ 0162243909334242.

Yakovleva, N., Flynn, A. (2004) Innovation and sustainability in the food system: A case of chicken production and consumption in the UK. Journal of Environmental Policy \& Planning, 6:3-4, 227-250. Recuperado de https:// www.tandfonline.com/doi/abs/10.1080/1523908042000344096

\section{Notas}

1 A partir del concepto de solidaridad de Émile Durkheim, Santos (2000) propone la idea de acontecer solidario para referirse a la realización común de tareas, aun cuando el proyecto no sea común. El acontecer solidario se presenta bajo tres formas en el territorio actual: además del jerárquico, el homólogo y el complementario. El acontecer homólogo es aquel en que la información motoriza un proceso de modernización y genera contigüidades funcionales, mientras que las relaciones de interdependencia entre las ciudades y el campo, consecuencia de las necesidades modernas de la producción, constituyen el acontecer complementario.

2 En la teoría de los circuitos de la economía urbana (Santos, 2008), la palabra circuito adquiere dos significaciones. Los circuitos espaciales de producción son definidos por la circulación de materia, es decir, "por las diferentes etapas por las cuales pasa un producto, desde el comienzo de la producción hasta llegar al consumo final" y "regulan el proceso productivo y aseguran la realización de capital" (Silveira, 2011, p. 6). Es preciso a su vez señalar que no podemos hablar de circuitos regionales de producción, ya que "el contexto de la producción y de la circulación no hace más que aumentar" (Silveira, 2011, p. 6). A su vez, ese movimiento es comandado por flujos no obligatoriamente materiales, es decir, capitales, informaciones, mensajes, órdenes, que constituyen los círculos de cooperación (Santos, 1996; Santos y Silveira, 2011). La otra acepción de los circuitos refiere a la posición estructural de los actores \#\# circuito superior e inferior de la economía urbana- y fue desarrollada en el párrafo anterior.

3 El área concentrada se caracteriza por la implantación más consolidada de datos de ciencia, técnica e información; el medio técnico-científico-informacional es entonces más continuo y más denso (Santos y Silveira, 2011). Se trata de un concepto construido con base en trabajos empíricos en Brasil. En la Argentina estamos proponiendo entender la porción de Ciudad Autónoma de Buenos Aires, las provincias de Buenos Aires, Córdoba y sur de Santa Fe como área concentrada.

4 Magdalena es una ciudad a $100 \mathrm{~km}$ de la ciudad de Buenos Aires, en la costa del Río de la Plata. Construidas en 1935, las instalaciones de Nestlé en Magdalena son de las más antiguas del grupo en el país. En la actualidad, además de productos lácteos para niños, el grupo realiza allí las mezclas y el envasado de los productos marca Maggi.

5 En la nota 2 el lector encontrará una descripción de los sistemas de conceptos principales que se articulan en la teoría de los circuitos de la economía urbana, entre ellos, el de círculos de cooperación.

6 BRF compró una firma avícola en la provincia de Córdoba (Avex) en octubre de 2011. La reciente venta de esta empresa por parte del grupo BRF forma parte de su proceso de reestructuración frente a la crisis inaugurada por la operación de investigación policial conocida como carne fraca (carne débil en su traducción al español). Los compradores fueron Granja Tres Arroyos y Fribel S.A.; Granja Tres Arroyos se quedaría con la faena de aves y Fribel con la parte de elaboración de margarina y aderezos.

7 Milton Santos (2000, p. 171) explica la convergencia de los momentos como "el conocimiento empírico de la simultaneidad de los acontecimientos y la comprensión de su significación interdependiente", los que, juntos, devienen factores determinantes de la realización histórica. Las nuevas técnicas, junto con la liberalización que resulta de la denominada desregulación económica, ofrecieron nuevos soportes a la circulación del dinero, lo que llevó a la tercera unicidad, la unicidad del motor; la emergencia de una plusvalía a nivel mundial (Santos, 2000).

8 El desarrollo genético avícola que se realiza en el país abarca desde la importación de abuelos, su reproducción, la cría y recría de padres y el engorde de los llamados pollitos $\mathrm{BB}$, que son finalmente comercializados. La genética y el crecimiento de los abuelos determinan la calidad de su descendencia, es decir, 7.000 pollitos BB (Palacios, 2003).

9 Desde la perspectiva de Milton Santos, existen puntos en el espacio que, separados unos de otros, aseguran el funcionamiento global de la sociedad y de la economía (Santos, 1999): "son vectores de una racionalidad superior y del 
discurso pragmático de los sectores hegemónicos, que crea un orden cotidiano obediente y disciplinado" (Santos, 2000, p. 240).

10 Aunque lo evalúan como necesario, en la actualidad no pueden afrontar el costo de las pruebas de mercado ni la contratación de consultorías de análisis de datos del mercado. Las otras áreas en las que contratan a otras empresas comprenden el circuito espacial de producción y ya no los círculos de cooperación: limpieza de tanques, mantenimiento de máquinas, frío, fumigación, lavado de ropa.

11 Las tarifas varían con la norma y pueden variar según la escala de producción; para cumplimentar con Global Gap hacia el año 2015 las empresas debían pagar una tarifa de U\$D 50.000 al año. 